\title{
How Many Kinds of Glue Hold the Social World Together?
}

\author{
BRIAN EPSTEIN
}

in Social Ontology and Social Cognition, Mattia Galloti and John Michael, eds.

Dordrecht: Springer, 2014

In recent years, theorists have debated how we introduce new social objects and kinds into the world. Searle, for instance, proposes that they are introduced by collective acceptance of a constitutive rule; Millikan and Elder that they are the products of reproduction processes; Thomasson that they result from creator intentions and subsequent intentional reproduction; and so on. In this chapter, I argue against the idea that there is a single generic method or set of requirements for doing so. Instead, there is a variety of what I call "anchoring schemas," or methods by which new social kinds are generated. Not only are social kinds a diverse lot, but the metaphysical explanation for their being the kinds they are is diverse as well. I explain the idea of anchoring and present examples of social kinds that are similar to one another but that are anchored in different ways. I also respond to Millikan's argument that there is only one kind of "glue" that is "sticky enough" for holding together kinds. I argue that no anchoring schema will work in all environments. It is a contingent matter which schemas are successful for anchoring new social kinds, and an anchoring schema need only be "sticky enough" for practical purposes in a given environment.

Among the most useful skills we have, as humans, is our ability to anchor new social kinds. We do this routinely. The furniture of today's world includes brands like Nike, Budweiser, and Blackberry; financial instruments like variable annuities, CDOs, and swaptions; technologies like screwdrivers, smartphones, and web services; dances like the lindy hop, jitterbug, and krump; textiles like gabardine, herringbone, and bouclé, subcultures like hipster, 
gopnik, and cybergoth; jobs like professor, President, barista, and climatologist; and so on. All of these are social creations, populating the world more richly and densely than it once was.

In this paper I will not concern myself with whether we genuinely introduce new social kinds into the world. I will take it for granted that we do, although this is a more loaded assumption than it might seem to be. My concern will be with how we do so. In particular, I argue against the idea that there is a single generic method or algorithm or set of requirements for anchoring new social kinds. Instead, there is a variety of "anchoring schemas," or methods by which new social kinds are generated. Not only are social kinds a diverse lot, but the metaphysical explanation for their being the kinds they are is diverse as well. My aim in this paper is to explain what this claim means, and put forward an intuitive case for it.

\section{What is anchoring? Dividing social ontology into two fields}

Although it is seldom recognized, social ontology divides into two separate fields of inquiry. First is what I will call the "grounding project." This is the inquiry into the grounds for the existence of a social object (such as a screwdriver or a hipster), the grounds for an object to have a social property (such as being a screwdriver or being a hipster), or to be a member of a social kind (such as screwdriver or hipster). ${ }^{1}$ This project is close to the one Frege initiated in The Foundations of Arithmetic of 1884. Following Frege, we might distinguish two different kinds of conditions associated with a property or kind: its instantiation conditions, and its identity conditions. The instantiation conditions are the conditions a given object needs to meet in order to have the property, or to be a member of the kind. ${ }^{2}$ The identity conditions are the

${ }^{1}$ Searle 1995, 2010 has popularized "institutional facts" as the central subject of social ontology. In his usage, however, the term is misleading. Many entities he discusses, such as dollars, boundaries, governments, etc., are social objects or kinds, not facts. And it is not clear that many of these involve institutions, in any of the standard senses of the notion.

${ }^{2}$ It is tedious to keep say "properties or kinds" or "having a given property or being a member of a given kind,” so I will mostly just speak of either properties or kinds, depending 
conditions under which two objects having that property are identical. If, for instance, an object $x$ satisfies the instantiation conditions for screwdriver, then it is a screwdriver. If both $x$ and $y$ are screwdrivers, and moreover satisfy the identity conditions for screwdriver, then they are the same screwdriver. The aim of the grounding project in social ontology is to give these sorts of conditions. ${ }^{3}$ For something to be a screwdriver, is it sufficient for it to be used to turn screws? Does it have to have a certain shape? Does it have to have been manufactured with a certain functional intention in mind? All these are questions in the Frege-style inquiry. They ask what it takes, what the conditions are, to ground the fact that something is a screwdriver.

The second inquiry I will call the "anchoring project." Though it has an equally long pedigree as the grounding project, it is a little less familiar. Suppose that a given social property or kind has such-and-such instantiation conditions, and such-and-such identity conditions. The anchoring project asks why are these the property or kind's instantiation and identity conditions? Or, to put the question slightly differently, why is this the property or kind that we have introduced or created? What have we done - or what facts are there in the world - that put a given property or kind, having these instantiation and identity conditions, in place? As I will term it, what facts anchor the property or kind? ${ }^{4}$

on which is most convenient. But the points about one can, in general, be extended to points about the other. Also, to be precise, Frege's analytic project was largely directed to terms and concepts, not properties. But roughly parallel distinctions apply.

${ }^{3}$ Strictly speaking, the identity conditions of a property are included among its instantiation conditions. (See Noonan 2009). Elsewhere, I have argued that despite this, we can nonetheless distinguish identity conditions from instantiation conditions (Epstein 2012b). Here I will mostly just speak of instantiation conditions, for brevity.

${ }^{4}$ It is my view that the anchors of a social kind are entirely distinct from the kind's instantiation conditions. Whatever puts the conditions in place for being an owner, or for being a screwdriver, or for being a hipster, is not itself among those instantiation conditions. This is a controversial stance. Some intuitive reasons for this claim come out in the next sections, but my principal aim is to clarify the notions of anchoring and 
A traditional approach to the anchoring project — for quite a few social properties and kinds, at least - comes from Hume: they are introduced by convention. ${ }^{5}$ For instance, Hume argues that the property being an owner is conventional. Many communities, for instance, once had the convention that the first person to occupy a piece of virgin territory is the owner of that property. In Hume's view, for a convention to be in place, within a community, is for members of the community to share certain beliefs about what will be to their mutual benefit. Thus Hume's answer to the question, What makes it the case that first occupants of a piece of land are its owners? is that we share various beliefs about how various practices involving first occupancy will be of mutual benefit. Those shared beliefs - the things that in Hume's view put in place a convention - are not the same as the conditions for someone to be an owner. For someone to be an owner of this sort is to be the first occupier of virgin territory. The shared beliefs about the benefits of the practice do not make any particular person an owner. Rather, the shared beliefs put in place or anchor the conditions for being an owner.

John Searle puts forward a somewhat different theory of anchoring, in his works on institutional facts. ${ }^{6}$ In Searle's view, properties like being an owner or being a dollar are anchored in certain collective attitudes we take, as a community. These collective attitudes are not just shared beliefs. According to Searlet, for the members of a community to collectively accept something, or collectively recognize something, is for each of the community members to have a "we-accept" or "we-recognize" attitude toward it. Thus according to Searle, what makes the first person to occupy a piece of territory its owner is this: we collective accept that people who are first-occupiers have the status

anchoring schemas. The rest of the paper does not depend on a rigid distinction between anchors and grounds: work on anchoring schemas is reasonably neutral on the question of whether the anchors of a social kind are among its instantiation conditions. For detailed treatment of this point, see Epstein (forthcoming).

${ }^{5}$ This tradition is actually a good bit older, but is most familiar from Hume.

${ }^{6}$ Searle 1995, 2010. 
and powers accorded to owners. The conditions for being an owner are anchored in collective acceptance.

Both Hume and Searle have unitary theories of how social properties and kinds are anchored. They both give a single account or schema for anchoring. In Hume's view, there is something special about convention, and in Searle's, something special about collective acceptance or recognition. But is there only one anchoring schema? What would it even mean for there to be more than one? To make sense of this, consider an analogous notion, widely discussed in a different field: the idea of word-introduction procedures in the philosophy of language.

\subsection{Descriptive semantics vs. foundational semantics}

In recent years, philosophers of language have distinguished two different fields within semantics. ${ }^{7}$ One is "descriptive semantics." This is the inquiry into what the meanings of words and sentences are. Some people hold, for instance, that the meaning of a proper name such as 'Plato' is just its referent, the person Plato. Others hold that the meaning of 'Plato' should be analyzed along the lines of "The person who wrote the Symposium, taught Aristotle, etc." ${ }^{8}$ Both of these theories of the meaning of a proper name are theories in the field of descriptive semantics. Also in descriptive semantics are theories of how the meanings of complex expressions are composed out of the meanings of words in combination with one another.

The second field is "foundational semantics." This is the inquiry into what makes it the case that words have the meanings they do. For proper names, for instance, many people endorse a "baptism-transmission" theory. ${ }^{9}$ This theory holds that the name 'Plato' has the meaning it does in virtue of its initial attachment to the person Plato a couple of thousand years ago, and the

\footnotetext{
${ }^{7}$ See Stalnaker 1997.

${ }^{8}$ See Kripke 1972/1980.

${ }^{9}$ Kripke, op cit.
} 
subsequent causal transmission of that name from person to person. A different theory holds that 'Plato' has the meaning it does in virtue of our current beliefs and communication practices.

The distinction between descriptive semantics and foundational semantics parallels the distinction I am advancing, between the grounding and anchoring projects in social ontology. Both descriptive semantics and foundational semantics are inquiries into the metaphysics of language. Descriptive semantics is the inquiry into certain key (perhaps essential) properties of words — namely, their semantic properties. ${ }^{10}$ Likewise, foundational semantics is not just an inquiry into historical happenstance, why a word happened to acquire those semantic properties. Instead, it is the inquiry into the facts that "put in place” the semantic facts. It looks for the metaphysical explanation for a word to have the meaning it does.

To be clear: although there are parallels between descriptive semantics and the grounding inquiry, we should take care to note that they are not the same thing. They cannot be the same, because there are many social properties we do not have words for. (There are, for instance, many properties that social scientists discover in their work.) Moreover, investigating the meaning of a word like 'screwdriver' is not the same thing as investigating the instantiation conditions of the social kind screwdriver. (For instance, a widely held theory of meaning takes the word 'screwdriver' simply to "refer directly" to that social kind. That is the entire descriptive semantics of the word 'screwdriver', and says nothing about the instantiation conditions of the kind screwdriver.) Equally, foundational semantics is not the same thing as the anchoring inquiry. Foundational semantics gives a metaphysical account of what puts in place a word, while the anchoring inquiry gives a metaphysical account of what facts put in place a social property.

Nevertheless, there are revealing parallels between the pairs of inquiries. In both domains — semantics and social ontology — we study a kind of tool. Words are linguistic tools, and social kinds are social tools. Yet these are not

${ }^{10}$ On the question of the essential properties of words, see Kaplan 1990 and Simchen 2012. 
just ordinary tools, but tools of a special sort: they are what we might call universal tools. Words are tools for expressing propositions, for saying things about actual and possible situations. (Words do other things as well, of course. ${ }^{11}$ But expressing ways the world is, was, or will be, or how it might be, is a key function words have.) Words are tools we apply to a universe of different situations - all the different possible ways the world might be, at any time, past, present, or future. They are not just tools for describing a restricted set of situations. A sentence like "A cat is on a mat" can be evaluated in any situation, any time, any world, even ones where English is not spoken.

Similarly, social properties and kinds are universal tools as well. They serve a variety of functions: we reference them when we recognize things, classify things in various situations, find and correct departures from norms, draw inductive inferences, and accomplish other practical matters. They too are applicable across a universe of different situations: we can look at any object whatever, in any situation, and assess whether that object is a member of the kind teacher, tire, hem, or hipster. That does not mean that social properties and kinds are not anchored in local contexts in the actual world. The kind hipster, for instance, is anchored by a range of idiosyncratic facts about our current society. But its potential instantiation is not limited to that current situation.

Because these fields investigate universal tools, both semantics and social ontology need to make a sharp distinction between two kinds of contexts: (1) the contexts in which the tools are employed, and (2) the contexts in which the tools are set up, or put in place. The reason for sharply separating these kinds of contexts is more easily seen in semantics. As I mentioned, when we evaluate the truth or falsity of a sentence like "A cat is on a mat," we might be interested in evaluating a situation ten thousand years ago, before English existed, or a million years ago, before any language was spoken. Or we might be interested in evaluating it in a world where there are no people at all. The descriptive semantics of the sentence "A cat is on a mat" is all that matters in evaluating

\footnotetext{
${ }^{11}$ See Austin 1962.
} 
the truth or falsity of that sentence, and the descriptive semantics is independent of the foundational facts that make that sentence mean what it does in English. When we evaluate the truth or falsity of "A cat is on a mat," we only need to look around the world for cats and mats, not for facts about what makes words have their meanings.

In semantics, that is, we distinguish the "contexts of evaluation" from the "contexts of assignment." Contexts of evaluation are the ones in which we evaluate expressions according to a fixed descriptive semantics. When we evaluate different situations in which the sentence "A cat is on a mat" may be true or false, we are considering contexts of evaluation. In evaluating the sentence, the foundational facts are irrelevant. When we browse around among contexts of evaluation, we take the descriptive semantics to be fixed as it is, even in the historical and possible situations where English does not exist. In ignoring the facts of foundational semantics, we allow the tool of linguistic expressions to be universally applicable.

Of course, there are also facts about the world that put those semantic facts in place. To investigate this - i.e., to do foundational semantics - we shift from contexts of evaluation to contexts of assignment. When we browse around contexts of assignment, we are not considering the facts that might make a sentence like "A cat is on a mat" true or false. Rather, we are browsing around the facts that make the word 'cat' have the meaning it does, the word 'on' have the meaning it does, and so on. In contexts of assignment, that is, we are not concerned about the evaluation of sentences. Instead, we consider the facts that put the descriptive semantics in place.

In social ontology, we likewise need to sharply separate two different contexts. Social properties and kinds are universal tools: they can be instantiated in any situation whatever. We can look back at ancient societies, and evaluate whether there are classes or castes, aristocrats or serfs. We can visit remote cultures, and inquire as to whether they have various forms of dance or song. We might look for baristas in the Ottoman Empire or in $17^{\text {th }}$ century England, and variable annuities among the ancient Egyptians. We might find that the Egyptians do not have variable annuities, but only proto- 
annuities. Or we might find that there is, in their context, an entity satisfying the instantiation conditions of being a variable annuity.

In evaluating whether a social property is instantiated in a given situation, we take those situations to be contexts of instantiation. A property like being President, or being the jitterbug, or being a cybergoth, applies to an object just in case it satisfies the relevant instantiation conditions. To evaluate whether an object has one or another of these properties, the anchors of those instantiation conditions are irrelevant.

However, we can also investigate the facts that anchor social properties and kinds, i.e., the facts in virtue of which those properties have the instantiation conditions they do. Like the investigation into foundational semantics, in this second inquiry we shift to a different context. Instead of browsing through contexts of instantiation, we browse through contexts of anchoring. In thinking about different contexts of anchoring, we are thinking about different ways various social properties can be anchored. In those contexts, we are not concerned with applying social properties - i.e., about whether a given object satisfies the instantiation conditions for a given social property. Rather, we might investigate which rules various people collectively accept, or which beliefs they have about mutual benefit. In considering contexts of anchoring, that is, we are concerned with the facts in virtue of which a social property is set up to be the particular universal tool it is, to be applied in any range of contexts of instantiation.

\subsection{Foundational schemas and anchoring schemas}

Foundational semantics investigates the facts that put words in place. One central part of this inquiry is the question of what procedures can be used for introducing words. (Another part of the inquiry is about how words are transmitted from person to person.) Much of the emphasis of foundational semantics has been on proper names. Different theorists have different accounts about what it takes to fix the reference of a proper name. One school argues that there is a single way for a proper name to be fixed in a language: the person introducing it needs to have a certain kind of acquaintance with the named object. A different school takes a broader view: reference fixing requires 
only unique identification of the referent, not acquaintance with it. Again, these are both metaphysical theories, explaining what grounds the fact that a proper name has the reference it does.

It is also possible that there is more than one schema for fixing the reference of a proper name. ${ }^{12}$ When we expand the inquiry beyond proper names, this is even more plausible: different words have their meanings in virtue of different kinds of facts. Some words may be defined, some words may be introduced by designating a sample by pointing at it, and some may be introduced by designating a sample by describing it. It may be that words of different types are introduced by different schemas. For instance, proper names might be introduced with one schema, and certain predicates with another. Or it may be that two different introduction schemas can be used to introduce words of several different types. It is the job of foundational semantics to characterize these schemas. These schemas are general methods or functions that describe which types of facts in the context of assignment metaphysically explain why words of a given type have the descriptive semantics they do.

A theory of anchoring, analogously, investigates the facts, in contexts of anchoring, that put social properties and kinds in place. Its aim is to characterize anchoring schemas. These are general methods or functions that describe which types of facts in the context of anchoring metaphysically explain why social kinds of a given types have the instantiation conditions they do. Hume's and Searle's respective theories propose different anchoring schemas, just as the acquaintance theorists and the latitudinarians propose different word-introduction schemas.

\section{Multiple anchoring schemas}

Is there just one overarching schema for anchoring social properties and kinds, or are there many? It is possible to trivialize this question. Suppose there are three different schemas. We could just combine them into one single schema, which is the disjunction of the three. Equally, if there is just one

${ }^{12}$ See Epstein 2008. 
schema, we could always break it up into sub-cases, turning one unified schema into several. Either of these, however, is just to play games. Putting aside tricks, this is a substantive question at the heart of social ontology: is the diverse furniture of the social world all explained in one way, by one generalized operation of our individual minds, or our collective minds, or our practices? Is the social world in its entirety a kind of projection of powers, by our minds, onto real substrates? ${ }^{13}$ Is it patterns of natural phenomena, to which we assign labels? ${ }^{14}$ Or is there more than one sort of account for social properties in general?

Consider the following three cases. All three draw on a key characteristic of many social kinds that hardly shows up in Searle's account: many kinds are what they are because of the properties of actual tokens. ${ }^{15}$ To explain why some kind $\mathrm{K}$ is the kind it is, we must look to actual objects in the world and the properties they have in common. We cannot only look to how we think about some set of objects, or how we cognize them. Instead, properties of sets of tokens of $\mathrm{K}$, and the relations among them, are part of the "glue" holding together $\mathrm{K}$ as a kind. ${ }^{16}$ The following three cases are not meant to be particularly unusual or distinctive. They are easily described cases of different sorts of social kinds, having histories that closely resemble one another.

\footnotetext{
${ }^{13}$ This is the sort of view Searle puts forward.

${ }^{14}$ As in Dennett 1991.

${ }^{15}$ Richard Boyd and Ruth Millikan highlight this in an exchange on "historical kinds" (Boyd 1999; Millikan 1999). See also Elder 2004.

${ }^{16}$ Ruth Millikan and Richard Boyd introduced this image in their exchange (Boyd and Millikan, op cit.). They also speak of the "ontological ground for the unity of a kind.” That terminology is not ideal, however, especially in light of the extensive recent literature on grounding, which uses the term 'ground' in a somewhat different way. Their respective theories in their exchange are, in part, theories of anchoring schemas. Theories, that is, about the sorts of things in the world — the histories, the causal mechanisms, the qualitative regularities - that set up the conditions for a disparate set of objects to be members of a kind.
} 
Case 1: The Aldino typeface

In the late $15^{\text {th }}$ century, Manutius Aldus commissioned Francesco Griffo, the Venetian punchcutter, to design a slanted typeface. It was attractive, highly legible, and its overlapping forms made efficient use of space on the page. Aldus named the new typeface 'Aldino'. Griffo's design was so successful that Aldus had the forms reproduced numerous times. He printed many volumes in the Aldino typeface.

Case 2: Pocket books

Aldus was also responsible for another innovation: the pocket book. In 1501, he began to print editions of Greek and Latin classics in small books with vellum covers. The first of these "libri portatiles" was an edition of the works of Virgil. Aldus went on to apply this format to dozens of books.

\section{Case 3: Italics}

Other printers took up the style of the Aldino typeface. Later versions refined and modified the slanted script typeface, and slanted script letterforms became widespread throughout Italy. Later on, this widespread letterform style was given the name 'italic'.

Each of these stories involves families of reproduced tokens - families that are not so different from one another. But in each case, a different sort of social kind is anchored. For each of these kinds, we can pursue both the grounding and the anchoring project. Both are rather complicated to work out in detail, but it is not too difficult to sketch plausible answers so long as we are content to leave them rough.

Consider the conditions a thing must satisfy in order to be an instance of the Aldino typeface. Like many kinds, members of Aldino can be any of a number of different sorts of entities. A particular set of marks on a page might be an instance of the Aldino typeface, or else a font description on a particular computer, or a set of metal type in a drawer might be. But to be an instance of Aldino is not just to have a particular pattern of letterforms. Instead, Aldino is plausibly a historical kind. For something to be an instance of that kind requires that it be a historical descendent of Griffo's original punches. If an identical 
letterform somehow occurred in nature, accidentally occurring, not a product of reproduction but just happenstance, it would not be an instance of Aldino. Likewise, if someone came up with an identical letterform from scratch, entirely causally disconnected from the history of reproduction of Griffo's punches, it also would not be an instance of Aldino. ${ }^{17}$ There are also qualitative conditions for something to be an instance of Aldino. Certain variations in letterforms are tolerated, but significant deviation from Griffo's forms suffices to preclude a set of marks, a font description, or a set of punches from being an instance. $^{18}$

The instantiation conditions for pocket book are somewhat different, despite the fact that Aldus reproduced the format much as he did the Aldino typeface. For an object to be an instance of a pocket book more likely involves a generic function, rather than being tied to one single historical family. Among the conditions for something to be a pocket book is plausibly that it have the function of being easily carried around in a pocket. We might understand this condition as a causal-role function, or else perhaps as a "Proper function": the function of being easily carried around in a pocket is part of the explanation for its having been produced. ${ }^{19}$ In either case, it has different sorts of instantiation conditions than Aldino does. For something to be an instance of Aldino requires that it be a member of a particular historical family, while for something to be an instance of pocket book does not. It is also plausible that there are qualitative characteristics an object must have, in order to be a pocket book, not just functional ones. A scroll that can be easily carried around in a pocket is not a pocket book.

The instantiation conditions for italic, in contrast, are purely qualitative. Today italics function more commonly for emphasis than for compactness, but whatever their function is, it has no bearing on whether a letterform is italic. All it takes for a letterform to be italic is for it to be written in a slanted script

\footnotetext{
${ }^{17}$ See Millikan 1984, Ch. 16.

18 See Elder, op cit.

${ }^{19}$ Millikan 1984
} 
style. Despite the similarity of its historical origins to Aldino, the conditions for a typeface to be italic are akin to those for a typeface to be "oblique." An oblique typeface is a slanted typeface, and an italic typeface is a slanted script typeface. (It is a peculiar quirk of the literature on artifacts and social kinds that current theories focus almost exclusively on functions, and so do not accommodate purely qualitative kinds, despite the fact that there is a good deal of evidence that many artifact kinds have purely qualitative instantiation conditions. ${ }^{20}$ )

Here we have three kinds with similar reproductive histories, and yet with three different sorts of instantiation conditions. Each of these kinds, in being anchored as it is, makes very different use of the tokens, their relation to one another, and other features of the environment. They are not held together by the same glue. Aldino is plausibly a historical kind in the sense of Millikan 1984 and 1999. Its dominant glue is the functional explanation for the proliferation of that particular family. Here is a rough way of depicting a schema of this sort: ${ }^{21}$

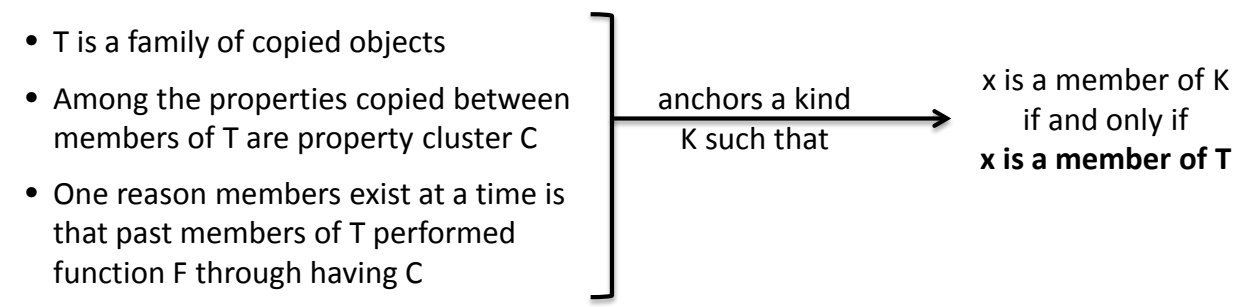

Figure 1. Schema with dominant ancestry / teleofunction

In this figure, the anchoring facts are listed on the left side, and the instantiation conditions of the kind are on the right. The figure represents a schema for anchoring a kind whose membership conditions are just that one is a member of a particular "reproductively established family." The family of copied objects is "glued together" by the fact that the reason for their being

\footnotetext{
${ }^{20}$ See Epstein 2012a.

${ }^{21}$ For a clear presentation of Millikan's picture see Godfrey-Smith 2004. I take some of the notation in this figure from that paper.
} 
copied as they are - and hence for being reproduced and hence members of $\mathrm{T}$ in the first place - is that $\mathrm{C}$ performs $\mathrm{F}$.

Pocket books are similar, except that they have been invented and reinvented many times, filling a fairly obvious "ecological niche.” The niche they fill is that people want to be able to easily carry their reading around with them. But as I mentioned, it is plausible that the kind pocket book is not restricted to one particular historically reproduced family. It applies as much to today's Penguin paperbacks as it does to Aldus's libri portatiles, regardless of the historical connectedness of these families. A plausible anchoring schema for pocket book might therefore be:

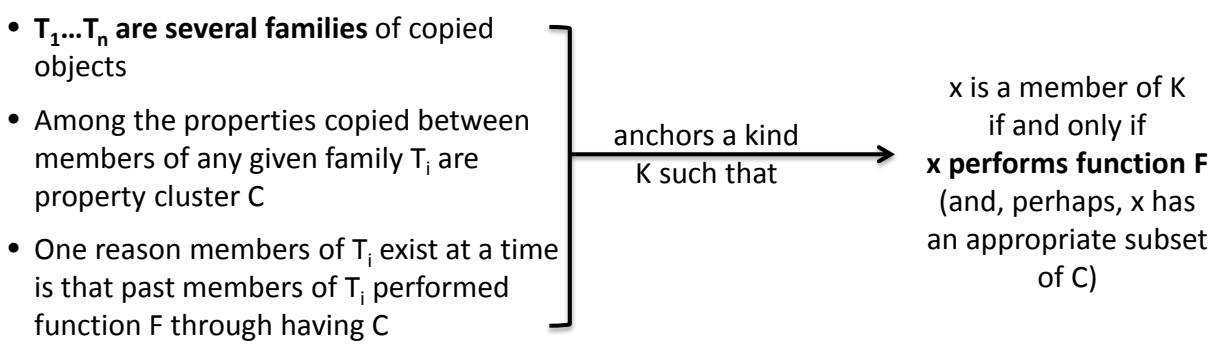

Figure 2. Schema with dominant generic function

The schema for anchoring italic also draws heavily on the existence of billions of easily recognized tokens, easily recognizable because of the simple qualitative contrast between their features and those of the billions of tokens of other letterforms that do not have those features (e.g., Roman characters). But italic is different from pocket book, in that there is no easily identified function at all, nor do we have a particular stake in associating a token with its actual ancestors, as opposed to its doppelgangers. Thus italic is plausibly anchored with a schema such as: 


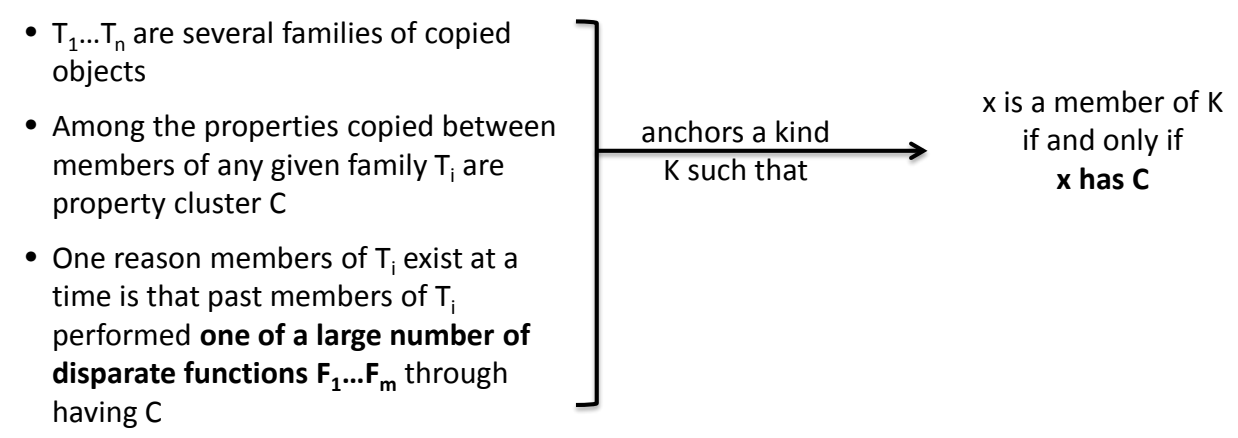

Figure 3. Qualitative dominance

These are only three of potentially a great number of anchoring schemas. There may be many ways to "glue together" a kind with qualitative instantiation conditions, or a functional kind. The aim of these examples is only to illustrate a few sorts of glue. Moreover, even if these three kinds have distinct "glues," still that does not entail that they have three different practical uses, as kinds. It may be, for instance, that all three kinds are useful for drawing inductions in a subfield of social science. ${ }^{22}$ Kinds anchored in several different ways may all be effective in that role. There may be several schemas, all of which are successful in practice, for anchoring kinds that serve in inductions. It is important to notice that serving in inductions is not a plausible criterion for gluing together a social kind. It is a plausible aim of social kinds, or role that social kinds play. But if a social kind is anchored in the right way then it is a kind even if it does not happen to work in inductions. ${ }^{23}$

Why do distinctive qualities "dominate" over functional characteristics, in the case of italic, or vice versa, in the case of pocket book? Why would being a member of a particular historical family dominate over serving a causal role

${ }^{22}$ Boyd and Millikan, op. cit. This is the role that many philosophers have insisted that social kinds play. My own inclination is that social kinds serve more diverse purposes, but for present purposes it is fine to take this more limited perspective.

${ }^{23}$ This is a (correct) feature of most all views. If, for instance, a kind is a Searle-style institution, or a Millikan-style teleofunctional kind, or a Boyd-style "homeostatic property cluster" kind, inductions over it may of course still fail. 
function, or vice versa? Sticking to the idea that a key role of kinds is to serve in drawing inductive inferences in the social sciences, it is easy to see how this can happen. When a large set of tokens is qualitatively distinct but functionally diverse, we respond more consistently to qualities than to functions. And when it is functionally unified, that functional unity is not only an outcome of our behavior, but influences it. If a practical purpose of social kinds is to figure into inductive inferences regarding human behavior, we should expect that certain kinds will be predominantly or even purely qualitative, while others will be functional, familial, or otherwise.

\section{How can these glues be sticky enough?}

Ruth Millikan has criticized certain liberal approaches to kinds, in particular ones that resemble the "dominant generic function" case I described above. Papineau 1992 and Macdonald 1992, for instance, present approaches to biological kinds that are less tied to particular historical families than is Millikan's. (Theirs is an approach to biological kinds, but similar arguments can be applied to the social case.) They argue that a functional kind can have multiple realizations in different reproduced families, when all the families are reproduced under similar selection pressures. For instance, there may be one generic selection pressure leading to different sorts of eye, or leading to different sorts of swimming traits. In such cases, eyes and swimmers may be biological kinds.

Millikan, for her part, does not deny that organisms under similar selection pressures can develop strikingly similar characteristics. However, she denies that that is sufficient to group these different realizations into a kind. In particular, she denies that such a "kind" would be sufficient to ground inductions:

Our question is not how a variety of different objects might come to exhibit the same functional property, but whether these objects would then form a proper natural kind over which inductions to further functional properties would be grounded. That a variety of objects all exhibit the same functionalist property for the same reason would not 
seem, by itself, to imply that they are alike in any other respects. ${ }^{24}$

One might think, Millikan points out, that realizations of a function in different families form a kind because they are selected to respond to the same pressures in a given ecological or evolutionary niche. But she objects:

This idea suffers from a misunderstanding of the role of an evolutionary niche. An evolutionary niche is not something that a species finds itself in and must then respond to, but something it creates for itself as it evolves by random mutation... Pairs of unrelated species in similar niches often do display some analogous characteristics, presumably for good reason, but occasional illuminating comparisons across species are not laws about the causal powers of niches. ${ }^{25}$

Millikan's argument is this. Consider members of two different families, both of which reproduce under similar selection pressures. That is not enough to ensure that the members in question have additional functional properties in common. In a single family, common properties will be copied because they realize the function. We can rely on this in order to be able to draw further inductions. But we cannot do the same when the families are different. This, then, is an example of an argument that some putative anchoring schema is not "sticky enough." It argues that the schemas proposed by Papineau and by Macdonald, as with the one I suggested in Figure 2, are insufficient to generate kinds over which we can draw inductive generalizations.

Millikan is surely right that we have to be careful about inferring similar characteristics from similar selection pressures. Her conclusion, however, is too general. Though we need to be careful, the fact that various families of organisms all solve a similar functional problem may indeed ground various sorts of unity. The fact that members of different species can swim, for instance, influences the ecosystem of predators that evolve to pursue swimmers, as opposed to non-swimmers. The presence of those predators, in turn, introduces new functional requirements on the entire class of swimmers.

\footnotetext{
${ }^{24}$ Millikan 1999, p. 59.

${ }^{25}$ Millikan 1999, p. 60.
} 
This establishes a link among functional properties, within that ecosystem: diverse families of swimmers are selected to exhibit new sets of functional properties in common. This, of course, is a simple example. But the linkages among functional properties arises even more easily in the social case, where we often care less about how objects perform their functions than we are about the fact that they do. So we pattern our behaviors accordingly: that is, according to what we care about or respond to. The fact that we respond to certain functional characteristics feeds back, in the social case, into objects having those functional characteristics also having other properties in common, functional and otherwise. Millikan is right that members of a kind like pocket book influence the ecological niches within which they are developed. But those feedback loops can serve to unify the niches across families as easily as they can divide them. Having descriptive properties in common does the same thing, in the social realm. Where there are billions of objects having some easily recognizable feature in common, that alone can ground cascades of social patterns.

That is only a quick response to Millikan's particular argument that this one sort of anchoring schema is not "sticky enough" to generate kinds. The real problem, however, is not with her particular argument, but with any argument of this form. It is too much to ask of any anchoring schema that it guarantee that kinds generated according to it will succeed at their intended role. No anchoring schema, for instance, will guarantee that kinds so-generated will underwrite successful inductive inferences. If the circumstances are infelicitous, inductions will fail even over members of a Millikan-style reproductively established family.

It cannot be known a priori that even Millikan's schema is successful at anchoring kinds, supposing it is. Instead, its success depends on contingencies of the circumstances. How sticky an anchoring glue is depends on how congenial the environment is to that sort of glue. A glue that will fail in hot climates may be excellent in cold ones. Similarly for an anchoring schema: being related in a given way may underwrite inductions in one "climate" and fail in another. This is as true of Millikan's schema as it is of any other. The 
ecosystems in our world happen to be regular enough that Millikan's schema generally works, to generate kinds that figure into inductive inferences. But that is a contingent matter. As a contingent matter, other anchoring schemas work as well. An anchoring schema need only be "sticky enough" to put in place tools that are practical. ${ }^{26}$

The contingency, practicality, and multiplicity of anchoring schemas does not mean the end of the anchoring inquiry, nor does it devalue that inquiry. We do not understand the nature of the social world if we do not understand anchoring, any more than we understand the nature of language without understanding what makes words have the meanings they do. In fact, these observations about anchoring schemas are only the first step in reconstructing a social ontology free of commitment to one secret sauce that makes the social world exist. Slogans like "for something to be a social object, it must be thought of as a social object," or "for something to be a social object, it must be created with some functional intention in mind," are widely repeated. But they are frankly incredible, given the immense diversity of the social world and the scanty understanding we have of it. An inquiry into the anchoring of the social world, I suggest, might better begin with broad investigation of diverse cases of social kinds, and investigation into the purposes social kinds may play. With these, we have a better hope of finding the various practical schemas by which social objects, properties, and kinds are set up, such that they — as a practical matter — tend to fill their roles and purposes.

\section{References}

Austin, J. L. (1962). How To Do Things With Words. Oxford: Clarendon Press.

${ }^{26}$ A similar point applies to reference-fixing procedures, I argue in Epstein 2008. 
Boyd, R. (1999). Kinds, Complexity and Multiple Realization: Comments on Millikan's "Historical Kinds and the Special Sciences". Philosophical Studies, 95(1-2), 67-98.

Dennett, D. (1991). Real Patterns. Journal of Philosophy, 88(1), 27-51.

Elder, C. (2004). Real Natures and Familiar Objects. Cambridge, Mass.: MIT Press.

Epstein, B. (2008). The Realpolitik of Reference. Pacific Philosophical Quarterly, 89, 1-20.

Epstein, B. (2012a). Review of Creations of the Mind, edited by Margolis and Laurence. Mind, 121(481), 200-04.

Epstein, B. (2012b). Sortals and Criteria of Identity. Analysis, 72(3), 474-78.

Epstein, B. (forthcoming). The Ant Trap: Rebuilding the Foundations of the Social Sciences.

Godfrey-Smith, P. (2004). A Modern History Theory of Functions. Noûs, 28, 344-62.

Kaplan, D. (1990). Words. Proceedings of the Aristotelian Society, 64, 93119.

Kripke, S. (1972/1980). Naming and Necessity. Cambridge: Harvard University Press.

Macdonald, G. (1992). Reduction and Evolutionary Biology. In D. Charles and K. Lennon (Eds.), Reduction, Explanation, and Realism (pp. 69-96). Oxford: Oxford University Press.

Millikan, R. G. (1984). Language, Thought, and Other Biological Categories: New Foundations for Realism. Cambridge: MIT Press.

Millikan, R. G. (1999). Historical Kinds and the "Special Sciences". Philosophical Studies, 95, 45-65. 
Noonan, H. (2009). What is a One-Level Criterion of Identity? Analysis, 69, 274-77.

Papineau, D. (1992). Irreducibility and Teleology. In D. Charles and K. Lennon (Eds.), Reduction, Explanation, and Realism (pp. 45-68). Oxford: Oxford University Press.

Searle, J. R. (1995). The Construction of Social Reality. New York: Free Press.

Searle, J. R. (2010). Making the Social World: The Structure of Human Civilization. Oxford: Oxford University Press.

Simchen, O. (2012). Necessity in Reference. In W. Kabasenche, M. O'Rourke, and M. Slater (Eds.), Reference and Referring (pp. 209-234). Cambridge: MIT Press.

Stalnaker, R. (1997). Reference and Necessity. In B. Hale and C. Wright (Eds.), A Companion to the Philosophy of Language (pp. 534-54).

Oxford: Blackwell. 